\title{
Measurements of the driving forces of bio-motors using the fluctuation theorem
}

\author{
Kumiko Hayashi $^{1}$, Mizue Tanigawara ${ }^{2,3}$ and Jun-ichi Kishikawa ${ }^{4}$ \\ ${ }^{1}$ Department of Applied Physics, School of Engineering, Tohoku University, Katahira, Aoba-ku, Miyagi 980-8577, Japan \\ ${ }^{2}$ School of Frontier Bioscience, Osaka University, Yamadaoka, Suita, Osaka 565-0871, Japan \\ ${ }^{3}$ Department of Applied Chemistry, School of Engineering, The University of Tokyo, Hongo, Bunkyo-ku, Tokyo 113-8656, Japan \\ ${ }^{4}$ Department of Molecular Biosciences, Faculty of Life Sciences, Kyoto Sangyo University, Motoyama, Kamigamo, Kita-ku, Kyoto 603- \\ 8555, Japan
}

Received January 30, 2012; accepted March 6, 2012

\begin{abstract}
The fluctuation theorem (FT), which is a recent achievement in non-equilibrium statistical mechanics, has been suggested to be useful for measuring the driving forces of motor proteins. As an example of this application, we performed single-molecule experiments on $F_{1}$-ATPase, which is a rotary motor protein, in which we measured its rotary torque by taking advantage of FT. Because fluctuation is inherent nature in biological small systems and because FT is a non-destructive force measurement method using fluctuation, it will be applied to a wide range of biological small systems in future.
\end{abstract}

Key words: motor protein, fluctuation, non-equilibrium statistical mechanics

\section{Fluctuation Theorem (FT)}

A macroscopic motor generates heat, $Q$, when energy to move the motor is injected. Defining an entropy production, $\sigma$, generated during a time interval, $\Delta t$, by $\sigma=Q / k_{\mathrm{B}} T$ where $k_{\mathrm{B}}$ is the Boltzmann constant and $T$ is the temperature of the environment, we find that $\sigma>0$. This empirical rule for a macroscopic system is called the second law of thermodynamics. However is $\sigma>0$ always observed for small systems

Corresponding author: Kumiko Hayashi, Department of applied physics, School of Engineering, Tohoku University, Katahira, Aoba-ku, Miyagi 980-8577, Japan.

e-mail: kumiko@camp.apph.tohoku.ac.jp such as motor proteins?

Before examining motor proteins, for simplicity, we considered a charged colloidal particle in water driven by an electric field (Fig. 1a). While motor proteins use energy from chemical reactions to move, the particle is driven by the force, $F$, exerted by the electric field. The position of the particle is denoted as $X(t)$. Heat, $Q$, generated during a time interval, $\Delta t$, is described by $Q=F \Delta X$, where $\Delta X=X(t+\Delta t)-$ $X(t)$. Because $X(t)$ fluctuates in time due to thermal noise, $Q$ has a diffierent value for each measurement. $Q>0$ when the particle moves in the same direction as that of the force while $Q<0$ when the particle moves against the force by being pushed by water molecules (which can happen if $\Delta t$ is small) (Fig. 1b). In the latter case $\sigma<0$. This apparent violation of the second law of thermodynamics is expressed by the fluctuation theorem (FT):

$$
\frac{P(\sigma)}{P(-\sigma)}=\mathrm{e}^{\sigma}
$$

where $P(\sigma)$ is the probability distribution of $\sigma$. Because Eq. (1) implies that the probability of $\sigma>0$ exponentially increases when the mean value of $\sigma$ becomes large, which is the case in a macroscopic system, Eq. (1) coincides with the second law of thermodynamics. FT was first advocated by Evans et al. in $1993^{1}$, and it was experimentally verified in a colloidal particle system in $2002^{2}$. Theoretically, many mathematical expressions of FT can be derived depending on system conditions ${ }^{3}$. Among them, the Crooks fluctuation theorem was experimentally verified in an RNA hairpin system $^{4}$. 


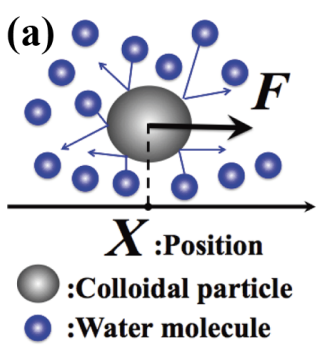

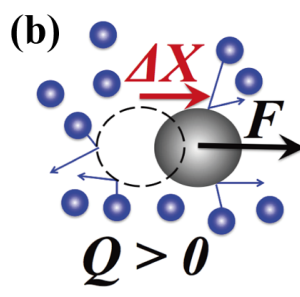

(c)

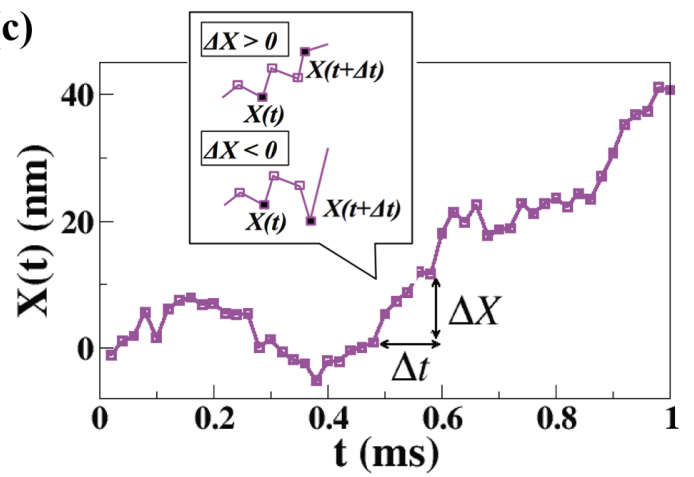

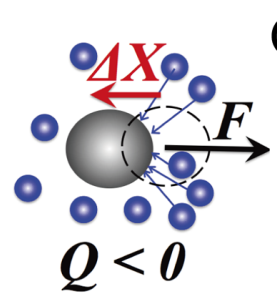
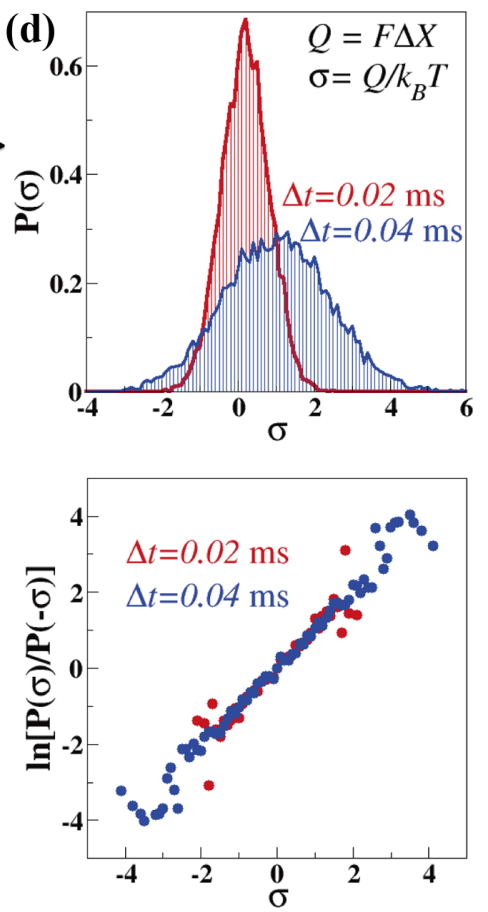

Figure 1 FT verified using simulation. (a) Schematic of a charged colloidal particle driven by an electric field. (b) Heat, $Q$, generated during $\Delta t$. (c) A sample time course, $X(t)$, obtained by the simulation. (d) (top) Probability distribution, $P(\sigma)$, of the entropy production, $\sigma$, in the cases $\Delta t=0.02 \mathrm{~ms}$ (red) and $\Delta t=0.04 \mathrm{~ms}$ (blue). (bottom) $\ln [P(\sigma) / P(-\sigma)]$ is plotted as a function of $\sigma$ in the cases $\Delta t=0.02 \mathrm{~ms}(\mathrm{red})$ and $\Delta t=0.04 \mathrm{~ms}(\mathrm{blue})$. It is seen that $\ln [P(\sigma) / P(-\sigma)]=\sigma$.

\section{Theory and Simulation}

To check Eq. (1), we performed a simulation of the colloidal particle system depicted in Figure 1a. Because the effect of the inertia is small in our colloidal particle system, the time evolution of $X(t)$ is described by the overdamped Langevin equation:

$$
\Gamma \frac{d X(t)}{d t}=F+\xi(t)
$$

where $\Gamma$ is the friction coefficient of the particle, and $\xi$ is Gaussian noise of the intensity $\sqrt{2 \Gamma k_{\mathrm{B}} T}$, which represents the effect of thermal noise. $\Gamma=6 \pi \eta a$ (Stokes law) where $\eta$ is the viscosity of water, and $a$ is the radius of the particle ${ }^{5}$. In Figure 1c, a sample time course of $X(t)$ is plotted for the case $a=1.5 \mu \mathrm{m}, T=25^{\circ} \mathrm{C}, \eta=0.89 \times 10^{-9} \mathrm{pNs} / \mathrm{nm}^{2}$, and $F=$ $1 \mathrm{pN}$. From $X(t)$, we calculated the probability distributions, $P(\sigma)$, of the entropy production, $\sigma=F \Delta X / k_{\mathrm{B}} T$ (where $\Delta X=$ $X(t+\Delta t)-X(t))$, for the cases $\Delta t=0.02 \mathrm{~ms}$ and $0.04 \mathrm{~ms}$ (Fig. $1 \mathrm{~d}$, left). In Figure 1d (right), $\ln [P(\sigma) / P(-\sigma)]$ is plotted as a function of $\sigma$. We can see that $\ln [P(\sigma) / P(-\sigma)]=\sigma$.

Next, we theoretically derive Eq. (1) using the model (2). Setting $X(t)=X_{1}$ and $X(t+\Delta t)=X_{2}$, the detailed balance condition in equilibrium ${ }^{5}$ is written as

$$
p_{\mathrm{eq}}\left(X_{1}\right) \operatorname{Tr}\left(X_{1} \rightarrow X_{2}\right)=p_{\mathrm{eq}}\left(X_{2}\right) \operatorname{Tr}\left(X_{2} \rightarrow X_{1}\right)
$$

bility from $X_{1}$ to $X_{2}$ (from $X_{2}$ to $\left.X_{1}\right) \cdot p_{\text {eq }}(X) \propto \mathrm{e}^{-E(X) / k_{\mathrm{B}} T}$ (the Boltzmann distribution), where $E$ represents the energy of the system.Then, Eq. (3) can be rewritten as

$$
\frac{\operatorname{Tr}\left(X_{1} \rightarrow X_{2}\right)}{\operatorname{Tr}\left(X_{2} \rightarrow X_{1}\right)}=\mathrm{e}^{-\Delta E / k_{\mathrm{B}} T}
$$

where $\Delta E=E\left(X_{2}\right)-E\left(X_{1}\right)$. In non-equilibrium in which $F \neq 0$ (Fig. 1a), $E(X)$ is modified into $E(X)-F X$ considering the energy injected into the system by the external driving force, $F$. Eq. (4) is then written as

$$
\frac{\operatorname{Tr}\left(X_{1} \rightarrow X_{2}\right)}{\operatorname{Tr}\left(X_{2} \rightarrow X_{1}\right)}=\mathrm{e}^{-(\Delta E-F \Delta X) / k_{\mathrm{B}} T}
$$

Eq. (5), which is the extended form in non-equilibrium of the detailed balance condition (Eq. (3)), is called the local detailed balance condition (LDB). See Hayashi and Sasa ${ }^{6}$ and Hayashi ${ }^{7}$ for more details on the LDB.

Because $\Delta E=0$ in our model (Eq. (2)), Eq. (5) is further rewritten as

$$
\frac{\operatorname{Tr}\left(X_{1} \rightarrow X_{2}\right)}{\operatorname{Tr}\left(X_{2} \rightarrow X_{1}\right)}=\mathrm{e}^{F \Delta X / k_{\mathrm{B}} T}
$$

When we consider the path $[X]=X_{1} \rightarrow X_{2} \rightarrow X_{3} \rightarrow \ldots X_{N}$, Eq. (6) is extended to

where $\operatorname{Tr}\left(X_{1} \rightarrow X_{2}\right)\left(\operatorname{Tr}\left(X_{2} \rightarrow X_{1}\right)\right)$ is the transition proba- 


$$
\frac{\boldsymbol{T R}([X])}{\boldsymbol{T R}([\tilde{X}])}=\mathrm{e}^{F \Delta X / k_{\mathrm{B}} T},
$$

where $[\tilde{X}]=X_{N} \rightarrow X_{N-1} \rightarrow \ldots \rightarrow X_{1}, \quad \Delta X=X_{N}-X_{1}$, $\boldsymbol{T R}([X])=\operatorname{Tr}\left(X_{1} \rightarrow X_{2}\right) \operatorname{Tr}\left(X_{2} \rightarrow X_{3}\right) \ldots \operatorname{Tr}\left(X_{N-1} \rightarrow X_{N}\right)$ and $\boldsymbol{T R}([\tilde{X}])=\operatorname{Tr}\left(X_{N} \rightarrow X_{N-1}\right) \operatorname{Tr}\left(X_{N-1} \rightarrow X_{N-2}\right) \ldots \operatorname{Tr}\left(X_{2} \rightarrow X_{1}\right)$ $\boldsymbol{T R}([X])$ and $\boldsymbol{T R}([\tilde{X}])$ are transition probabilities for the paths $[X]$ and $[\tilde{X}]$, respectively. Then the probability distribution of the entropy production, $\sigma=F \Delta X / k_{\mathrm{B}} T$, is defined by

$$
P(\sigma)=\int d[X] \delta\left(\sigma-F \Delta X / k_{\mathrm{B}} T\right) \boldsymbol{T R}([X]) .
$$

With Eq. (7), Eq. (8) is rewritten as

$$
\begin{aligned}
P(\sigma) & =\int d[X] \delta\left(\sigma-F \Delta X / k_{\mathrm{B}} T\right) \mathrm{e}^{F \Delta X / k_{\mathrm{B}} T} \boldsymbol{T R}([\tilde{X}]) \\
& =\int d[\tilde{X}] \delta\left(\sigma+F \Delta \tilde{X} / k_{\mathrm{B}} T\right) \mathrm{e}^{-F \Delta \tilde{X} / k_{\mathrm{B}} T} \boldsymbol{T R}([\tilde{X}]) \\
& =\int d[X] \delta\left(S+F \Delta X / k_{\mathrm{B}} T\right) \mathrm{e}^{-F \Delta X / k_{\mathrm{B}} T} \boldsymbol{T R}([X]) \\
& =P(-\sigma) \mathrm{e}^{\sigma},
\end{aligned}
$$

where $\Delta \tilde{X}=-\Delta X$. Eq. (9) represents Eq. (1). Physical interpretations of Eq. (9) seem difficult because Eq. (9) is obtained formally from the mathematical identity transformation using the LDB (Eq. (5)). In this paper, we do not consider the physical interpretations of Eq. (9), but consider how to use Eq. (9) in single-molecule experiments. See Hayashi and $\mathrm{Sasa}^{6}$ for details, and Hayashi ${ }^{7}$ for a brief summary on Eq. (9).

\section{Using FT to Measure the Driving Forces of Bio-motors}

In the case of a linear motor protein that moves in one direction, the driving force, $F$, and thermal noise act on a cargo (Fig. 2, left). For this model, we can derive the following expression of the fluctuation theorem:

$$
\ln [P(\Delta X) / P(-\Delta X)]=F \Delta X / k_{\mathrm{B}} T,
$$

where $X(t)$ is the position of the cargo, $\Delta X=X(t+\Delta t)-X(t)$ and, $P(\Delta X)$ is the probability distribution of $\Delta X$. We use $\Delta X$ instead of $\sigma$ in Eq. (9) to derive Eq. (10). In the case of a rotary motor protein that rotates in one direction, the rotary torque, $N$, and thermal noise act on a bead (Fig. 2, right). Similarly to Eq. (10), we obtain,

$$
\ln [P(\Delta \theta) / P(-\Delta \theta)]=N \Delta \theta / k_{\mathrm{B}} T,
$$

where $\theta(t)$ is the rotary angle of the bead, $\Delta \theta=\theta(t+\Delta t)-\theta(t)$, and $P(\Delta \theta)$ is the probability distribution of $\Delta \theta$. In Eqs. (10) and (11), we assume that $F$ and $N$ are constant. Such an assumption is valid when the variations in a driving force are much smaller than the mean value of the driving force.

We can obtain $X(t)(\theta(t))$ from single-molecule experiments. Measuring $P(\Delta X) \quad(P(\Delta \theta))$, when we plot

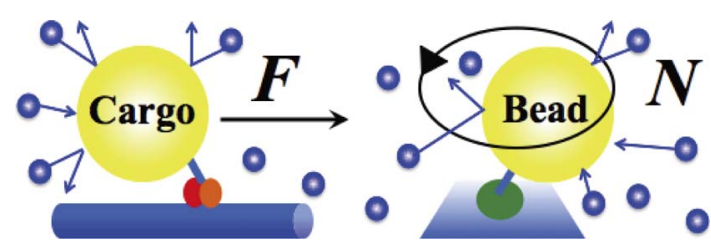

Figure 2 Driving forces of motor proteins. (left) Schematic of a linear motor protein. A driving force, $F$, exerted by a motor and thermal noise act on a cargo. (right) Schematic of a rotary motor protein. A rotary torque, $N$, exerted by a motor and thermal noise act on a bead.

$\ln [P(\Delta X) / P(-\Delta X)] \quad(\ln [P(\Delta \theta) / P(-\Delta \theta)])$ as a function of $\Delta X / k_{\mathrm{B}} T\left(\Delta \theta / k_{\mathrm{B}} T\right)$, the slope of the graph corresponds to $F(N)$. See the next section for an example.

\section{Application of Eq. (11) to $\mathrm{F}_{1}$-ATPase}

$\mathrm{F}_{1}$ is a rotary motor protein and a part of $\mathrm{F}_{0} \mathrm{~F}_{1}$ ATPase/synthase ${ }^{8-14}$. The minimum complex that can act as a motor is the $\alpha_{3} \beta_{3} \gamma$ subcomplex, in which the $\gamma$ subunit rotates in the $\alpha_{3} \beta_{3}$ ring (Fig. 5a, top, left) upon ATP hydrolysis. The three catalytic $\beta$ subunits hydrolyze ATP sequentially and cooperatively. Three ATP molecules are hydrolyzed per turn, or in other words, the free energy obtained from the hydrolysis of a single ATP molecule is used for a $120^{\circ}$ rotation. The conformations of the $\beta$ subunits change as the elementary chemical steps, such as the ATP binding, the ATP hydrolysis (cleavage of the covalent bond), and the product releases (ADP and inorganic phosphate) proceed (Fig. 3a). The coordinated push-pull motion of the Cterminal domains of the $\beta$ subunits produces torque for the $\gamma$ subunit to rotate ${ }^{9,10}$.

In our single-molecule assay (see Methods in Hayashi et $\left.a l .{ }^{11}\right)$, the rotation of the $\gamma$ subunit was observed as the rotation of a bead attached to it (Fig. 3b) because the size of the $\gamma$ subunit itself is too small $(\sim 2 \mathrm{~nm})$ for its rotation to be observed directly under an optical microscope. The rotational angle, $\theta(t)$, was calculated from the recorded images of the bead (Figs. 3c and 3d). In Figure 3c, $\theta(t)$ is plotted for the cases of $1 \mathrm{mM}$ ATP and $100 \mathrm{nM}$ ATP. While the rotation was continuous for $1 \mathrm{mM}$ ATP, it became stepwise for $100 \mathrm{nM}$ ATP, pausing every $120^{\circ}$. The ATP-binding dwell (the time that a catalytic site waits for ATP binding) is very short at high [ATP] $(<0.1 \mathrm{~ms}$ at $1 \mathrm{mM})$, and the time constants of the dwells for ATP hydrolysis and that for the product releases are also short (about $1 \mathrm{~ms}$ independent of [ATP] for the wild-type $F_{1}$ ). The rotation appears continuous when the response time of a bead is longer than these time periods.

In previous studies ${ }^{12-15}$, the rotary torque, $N$, was estimated using the equation

$$
N=\Gamma \omega,
$$

where $\omega$ and $\Gamma$ are the mean angular velocity and friction coefficient of a probe (e.g. a bead), respectively. From the 
(a)

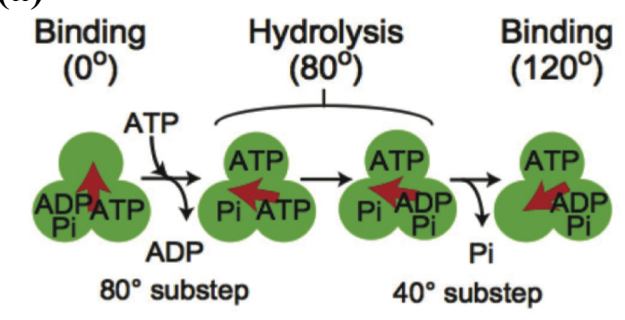

(b)

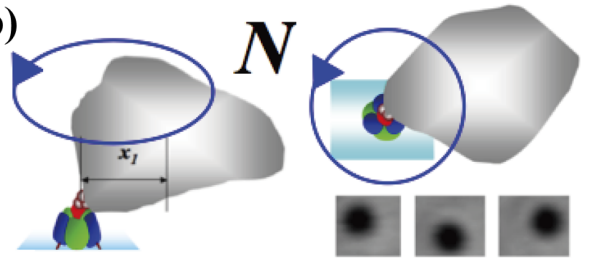

(c)

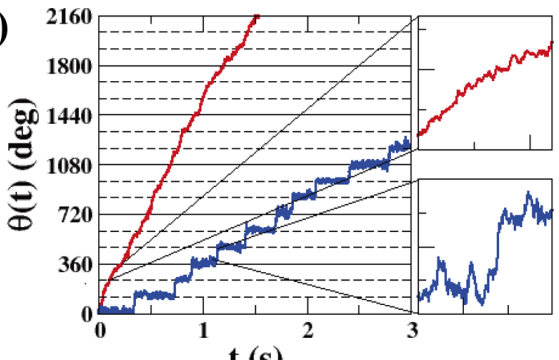

(d)

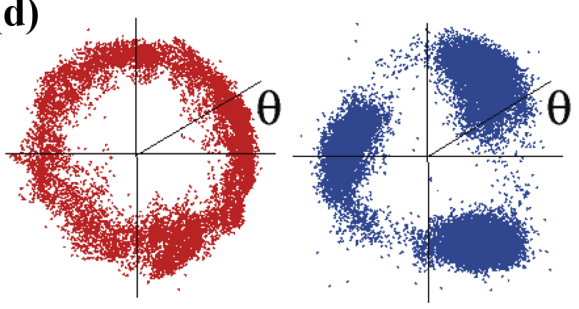

Figure 3 Rotary motor protein, $\mathrm{F}_{1}$. (a) Reaction scheme of $\mathrm{F}_{1}$. The green circles and the red arrow represent the $\beta$ subunits and $\gamma$ ubunit of $\mathrm{F}_{1}$, respectively. $\mathrm{F}_{1}$ performs a $120^{\circ}$ step rotation upon ATP hydrolysis consisting of $80^{\circ}$ and $40^{\circ}$ substeps. (b) Schematic diagrams of our experimental system (not to scale). The rotation of $\mathrm{F}_{1}$ is probed by an irregularly shaped magnetic bead (see Methods in Hayashi et al. ${ }^{11}$ for this irregular shape). The size of $F_{1}$ is about $10 \mathrm{~nm}$ and the size of the bead is about $300-500 \mathrm{~nm}$. (c) ATP-driven rotations of $F_{1}$ probed by the magnetic beads at $1 \mathrm{mM}$ ATP (red) and $100 \mathrm{nM}$ ATP (blue). The recording rate was $2000 \mathrm{fps}$. (d) The center of mass of the bead was calculated from the recorded images for the case of $1 \mathrm{mM}$ ATP (red) and $100 \mathrm{nM}$ ATP (blue).

calculation of fluid mechanics, the functional forms of $\Gamma$ are known as

$$
\begin{aligned}
& \Gamma_{\mathrm{s}}=8 \pi \eta a^{3}+6 \pi \eta a x_{1}^{2} \text { for an a single bead, } \\
& \Gamma_{\mathrm{d}}=16 \pi \eta a^{3}+6 \pi \eta a x_{1}^{2}+6 \pi \eta a x_{2}^{2} \text { for a duplex of beads, }
\end{aligned}
$$

where $a$ and $x_{i}(i=1,2)$ are the radius and rotation radius of each bead, and $\eta$ is the viscosity of water $\left(\eta=8.9 \times 10^{-10}\right.$ $\mathrm{pNs} / \mathrm{nm}^{2}$ at $25^{\circ} \mathrm{C}$ ). Note that Eq. (13) is derived under the assumption that the rotation of a bead or a duplex of beads occurs in bulk. This causes inaccuracy in estimating the friction coefficients of beads in the case of the single-molecule experiment on $\mathrm{F}_{1}{ }^{15}$. Because $\mathrm{F}_{1}$ attaches a glass slide and rotates the beads near the glass surface (Fig. 2b), an interaction between the beads and the glass makes the estimation using Eq. (13) diffierent from the real value. In fact, the real value of $\Gamma$ is larger than the value estimated by Eq. (13). In single-molecule experiments in general, it is difficult to obtain accurate values of the friction coefficients of probes, because the probe sizes are distributed and their exact shapes are unknown, and because the motion of probes is often observed close to the surface of a glass slide, where motor proteins attach, rather than in bulk. In fact, the difficulty in estimating $\Gamma$ is a common problem for force measurements of biological motors such as a bacterial flagella ${ }^{16}$ which rotates a bead near the surface of a cell, and an RNA polymerase ${ }^{17}$ which moves near the surface of a glass slide. To overcome this problem, we use FT (Eqs. (10) and (11)) that can estimate driving forces without using the value of $\Gamma$.

For the continuous rotation of $\mathrm{F}_{1}$ probed by a bead (Fig. 4a, top), $P(\Delta \theta)$ and $\ln [P(\Delta \theta) / P(-\Delta \theta)]$ are plotted for the cases $\Delta t=2.5-10 \mathrm{~ms}$ (Fig. $4 \mathrm{a}$, bottom). The slopes of the graphs in Figure 4a (bottom, right) are almost the same for all cases $\Delta t=2.5-10 \mathrm{~ms}$, and the value of the slope in the case $\Delta t=10 \mathrm{~ms}$ is $38 \mathrm{pNnm}$, which corresponds to $N$ according to Eq. (11). This value coincides with previous observations $^{12,13}$. See Hayashi et al. ${ }^{11}$ for details about the application of Eq. (11), e.g., the recording rate dependence, the bead-size dependence, $\Delta t$ dependence and the comparison between Eqs. (11) and (12).

For the wild-type $\mathrm{F}_{1}$, the rotation became stepwise at $100 \mathrm{nM}$ ATP (Figs. $3 \mathrm{c}$ and $4 \mathrm{~b}$, top). In this case, $\Delta \theta$ was calculated using the $120^{\circ}$ steps encircled in red (Fig. $4 \mathrm{~b}$, top). In Figure 4b (bottom), $P(\Delta \theta)$ and $\ln [P(\Delta \theta) / P(-\Delta \theta)]$ are plotted for the cases $\Delta t=0.5-2.5 \mathrm{~ms}$. From the slope of the graph, we obtained $40 \mathrm{pNnm}$. Note that the values of $\Delta t$ are smaller than those used at $1 \mathrm{mM}$ ATP because the $P(\Delta \theta)$ for $\Delta t$ larger than $2.5 \mathrm{~ms}$ was hard to measure precisely for the stepping rotations due to a small number of samples. See Hayashi et al. ${ }^{11}$ for the stepping rotations of the mutant $\mathrm{F}_{1}$ $(\beta \mathrm{E} 190 \mathrm{D})$, whose torque was also about $40 \mathrm{pNnm}$.

\section{Application of (11) to $V_{1}$-ATPase}

We applied FT (Eq. (11)) to another rotary motor $\mathrm{V}_{1}$, which is a part of $\mathrm{V}_{\mathrm{o}} \mathrm{V}_{1}$-ATPase ${ }^{18}$ (see also Supplementary Material of Hayashi et al. ${ }^{11}$ for the rotation assay of $\mathrm{V}_{1}$ ). In $\mathrm{V}_{1}$, the $\mathrm{D}$ subunit rotates in the $\mathrm{A}_{3} \mathrm{~B}_{3}$ ring and $120^{\circ}$ steps are observed at low [ATP] (in our case $[\mathrm{ATP}]=10 \mu \mathrm{M}$ ). Using Eq. (11), we obtained $33 \pm 2.2 \mathrm{pNnm}$ as the rotary torque of $\mathrm{V}_{1}$, which was smaller than the rotary torque of the wildtype $\mathrm{F}_{1}(38 \pm 2.7 \mathrm{pNnm})^{11}$. 
(a)
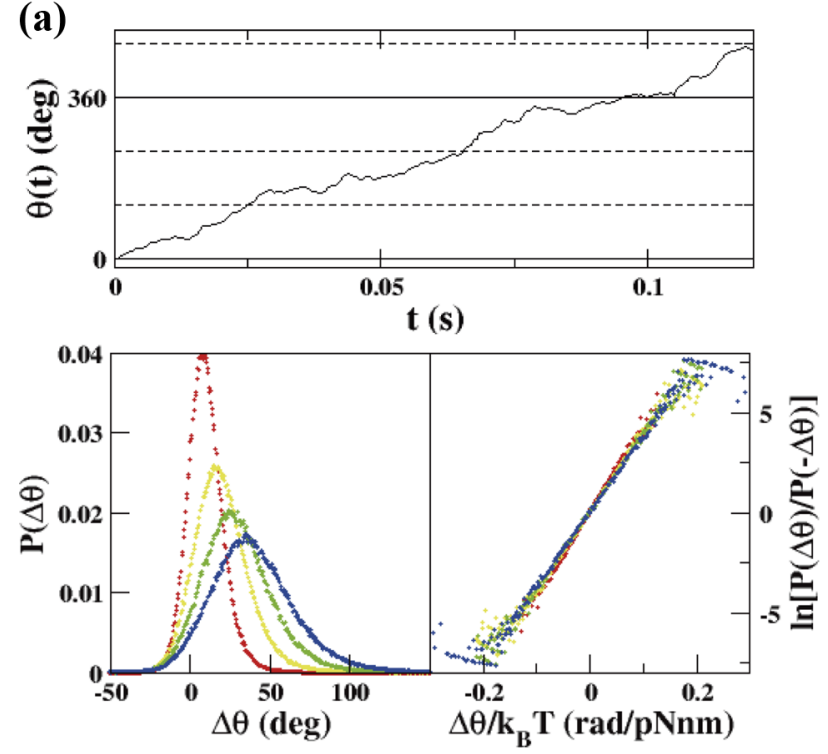

(b)
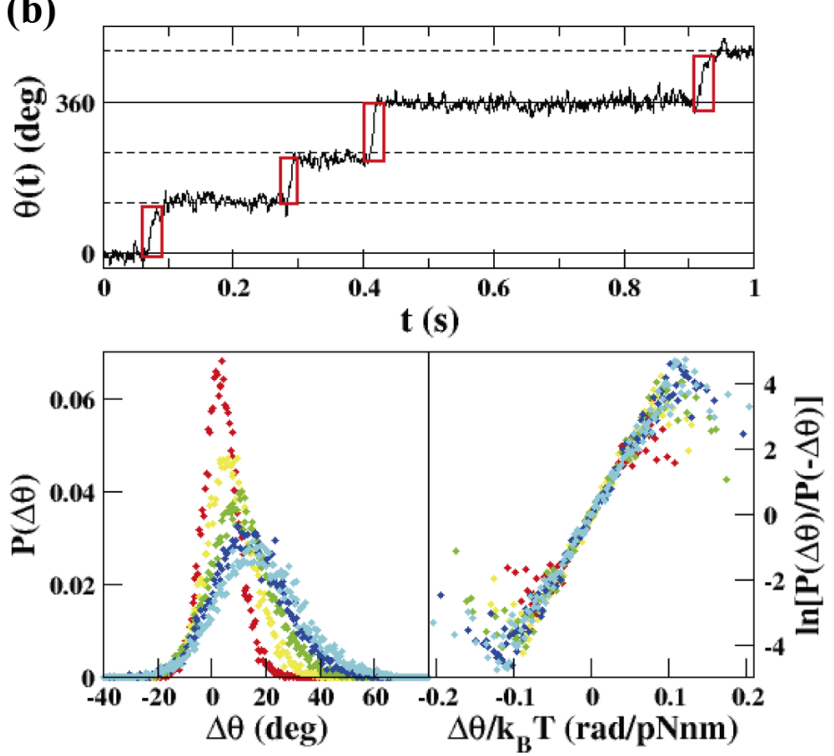

Figure 4 FT applied to the wild-type $\mathrm{F}_{1}$. (a) (top) ATP-driven rotation of $\mathrm{F}_{1}$ at $1 \mathrm{mM}$ ATP. (bottom, left) Probability distribution, $P(\Delta \theta)$, for the cases $\Delta t=2.5 \mathrm{~ms}$ (red), $5.0 \mathrm{~ms}$ (yellow), $7.5 \mathrm{~ms}$ (green) and $10 \mathrm{~ms}$ (blue) for the particular $\mathrm{F}_{1}$ probed by the magnetic bead. (bottom, right) $\ln [P(\Delta \theta) / P(-\Delta \theta)]$ as a function of $\Delta \theta / k_{\mathrm{B}} T$. The slope was $38 \mathrm{pNnm}$ in the case $\Delta t=10 \mathrm{~ms}$. The recording rate was $2000 \mathrm{fps}$. (b) (top) ATP-driven rotation of $\mathrm{F}_{1}$ at $100 \mathrm{nM}$ ATP. We analyzed the $\theta(t)$ encircled in red (we used about 100-300 steps). The steps were determined by eye. (bottom, left) Probability distributions, $P(\Delta \theta)$, for the cases $\Delta t=0.5 \mathrm{~ms}$ (red), $1.0 \mathrm{~ms}$ (yellow), $1.5 \mathrm{~ms}$ (green), $2.0 \mathrm{~ms}$ (blue), and $2.5 \mathrm{~ms}$ (aqua) for the particular $\mathrm{F}_{1}$ probed by the magnetic bead. (bottom, right) $\ln [P(\Delta \theta) / P(-\Delta \theta)]$ as a function of $\Delta \theta / k_{\mathrm{B}} T$. The slope was $40 \mathrm{pNnm}$ in the case $\Delta t=2.5 \mathrm{~ms}$. The recording rate was $2000 \mathrm{fps}$.
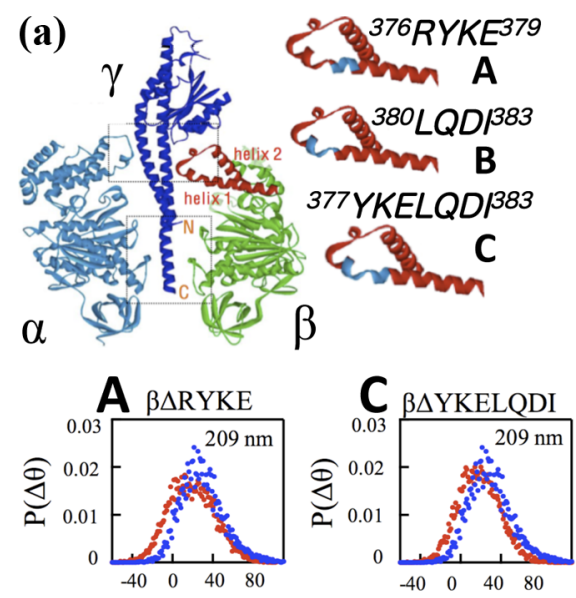

$\Delta \theta(\mathrm{deg})$

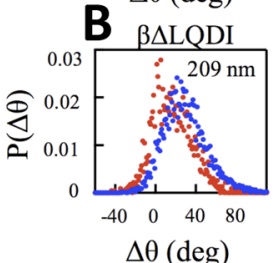

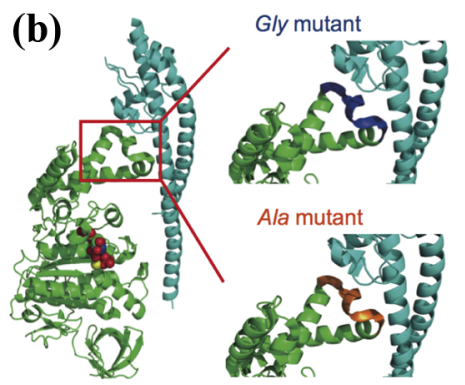

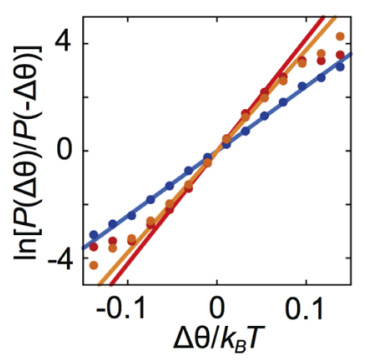

Wild-type $38 \mathrm{pNnm}$ Gly mutant $24 \mathrm{pNnm}$ Ala mutant $37 \mathrm{pNnm}$
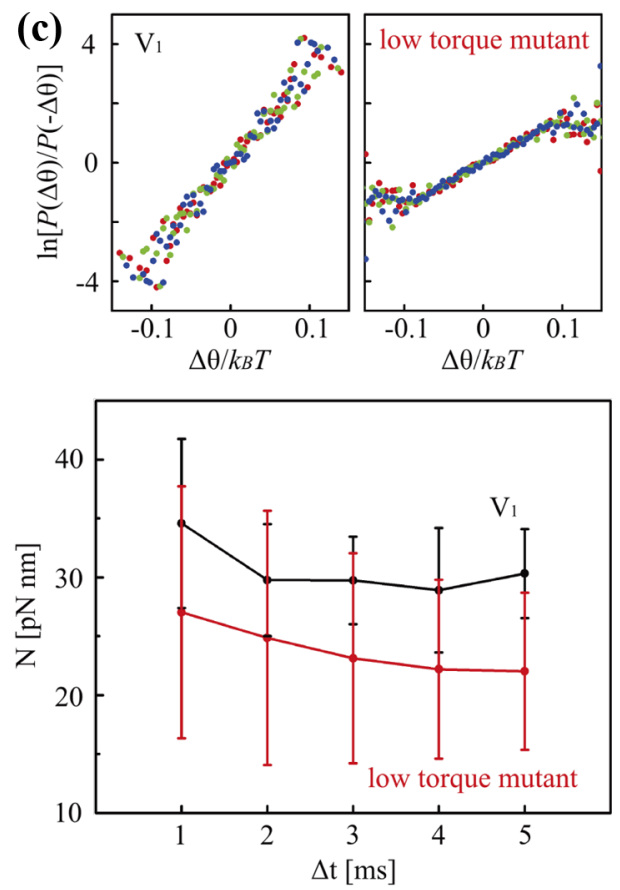

Figure 5 FT applied to mutants $\mathrm{F}_{1}$ and $\mathrm{V}_{1}$. (a) (top) The structures of $\alpha, \beta$ and $\gamma$ subunits of the $\mathrm{F}_{1}$. Truncated sequences are shown in blue ${ }^{19}$. (bottom) $P(\Delta \theta)$ for the wild-type $\mathrm{F}_{1}$ (blue) and the mutants (red) probed by 209 -nm sized duplex beads in the case $\Delta t=4$-ms at $[\mathrm{ATP}]=2 \mathrm{mM}$. Torque was calculated from $P(\Delta \theta)$ using Eq. (11). (b) (top) The structures of $\beta$ and $\gamma$ subunits of the $\mathrm{F}_{1}$. Residues replaced by glycine (Gly mutant) and alanine (Ala mutant) are shown in blue and orange, respectively.(bottom) $\ln [P(\Delta \theta) / P(-\Delta \theta)]$ as a function of $\Delta \theta / k_{\mathrm{B}} T$ for the stepping rotations probed by magnetic beads in the case $\Delta t=5 \mathrm{~ms}$ at $[\mathrm{ATP} \gamma \mathrm{S}]=1 \mathrm{mM}$. The recording rate was $1000 \mathrm{fps}$. (c) (top) Comparison between $\mathrm{V}_{1}$ and a rotormodulated mutant of $\ln [P(\Delta \theta) / P(-\Delta \theta)]$ as a function of $\Delta \theta / k_{\mathrm{B}} T$ for the stepping rotations probed by magnetic beads in the cases $\Delta t=3 \mathrm{~ms}(\mathrm{red})$, $\Delta t=4 \mathrm{~ms}$ (green), and $\Delta t=5 \mathrm{~ms}$ (blue) at $[\mathrm{ATP}]=10 \mu \mathrm{M}$. The recording rate was $1000 \mathrm{fps}$. (bottom) $\Delta t$ dependence of $N$ measured using Eq. (11) for $\mathrm{V}_{1}$ (black) and the mutant $\mathrm{V}_{1}$ (red). Results are the average from 5 molecules (black) and 4 molecules (red), respectively. 


\section{Further Applications to Lower-Torque Mutants}

To obtain lower-torque mutants, contact regions in $F_{1}$ between the catalytic $\beta$ subunits and the rotor $\gamma$ subunit were mutated. The torque generation of these mutants $F_{1}$ was investigated (Fig. 5a, top) ${ }^{19}$. By the analysis of FT (Eq. (11)), the torque value is directly obtained from the fluctuation of a rotating bead, without needing to know the value of the friction coefficient, which requires the exact size of the bead, the rotational radius, and the viscosity of the medium near the surface of a glass slide ${ }^{19}$. Taking advantage of Eq. (11), the torques of the mutants $F_{1}$ were accurately compared with that of the wild-type $\mathrm{F}_{1}$ (Fig. 5a, bottom). We also investigated other lower-torque mutants of $\mathrm{F}_{1}$ (Fig. 5b) and a lower-torque mutant of $\mathrm{V}_{1}$ (Fig. 5c) by using Eq. (11).

\section{Discussion}

Besides the rotary motor proteins studied here, FT (Eqs. (10) and (11)) may be applied to other biological systems, including bacterial flagella and linear motor proteins such as kinesins and myosins. We hope that FT, which is a nondestructive force measurement method using fluctuation, will be applied to a wide range of biological systems in future.

When we consider applying FT to more complex biological systems, we need to note the limitations of using Eqs. (10) and (11). The temperature of the environment, $T$, appears in these equations because we assumed that fluctuation in the system were attributed to thermal noise. The application of FT to cell motion, which is affected by spontaneous fluctuation of a cell in addition to thermal noise, explains how the spontaneous fluctuation causes problems to use Eqs. (10) and (11). See Hayashi and Takagi ${ }^{20}$ for details.

\section{Acknowledgments}

This work was supported by Grant-in-Aid for Scientific Research from the MEXT (No. 23107703) to K.H., and by Honjo International Scholarship Foundation to M.T. We thank Dr. R. Watanabe for the analysis of the mutant $F_{1}$, and A. Nakanishi for the experiment on the mutant $V_{1}$.

\section{References}

1. Evans, D. J., Cohen, E. G. D. \& Morriss, G. P. Probability of second law violations in shearing steady states. Phys. Rev. Lett. 71, 2401-2404 (1993).

2. Wang, G. M., Sevick, E.M., Mittag, E., Searles, D. J. \& Evans, D. J. Experimental demonstration of violations of the second law of thermodynamics for small systems and short time scales. Phys. Rev. Lett. 89, 050601 (2002).

3. Ciliberto, S., Joubaud, S. \& Petrosyan, A. Fluctuation in out of equilibrium systems: from theory to experiment. J. Stat. Mech. P12003 (2010).

4. Collin, D., Ritort, F., Jarzynski, C., Smith, S. B., Tinoco Jr, I. $\&$ Bustamante, C. Verification of the Crook fluctuation theorem and recovery of RNA folding free energies. Nature $\mathbf{4 3 7}$, 231-234 (2005).

5. Howard, J. Mechanics of Motor Proteins and the Cytoskeleton (Sinauer Associates Inc, 2001).

6. Hayashi, K. \& Sasa, S. Linear response theory in stochastic many-body systems revisited. Physica A 370, 407-429 (2006).

7. Hayashi, K. Fluctuation theorem applied to bio-motors (review in Japanese). Seibutsu Butsuri 51, 188-189 (2011).

8. Noji, H., Yasuda, R., Yoshida, M. \& Kinosita Jr, K. Direct observation of the rotation of $\mathrm{F}_{1}$-ATPase. Nature 386, 299302 (1997).

9. Wang, H. \& Oster, G. Energy transduction in the $\mathrm{F}_{1}$ motor of ATP systhase. Nature 396, 279-282 (1998)

10. Masaike, T., Koyama-Horibe, F., Oiwa, K., Yoshida, M. \& Nishizaka, T. Cooperative threestep motions in catalytic subunits of $\mathrm{F}_{1}$-ATPase correlate with $80^{\circ}$ and $40^{\circ}$ substep rotation. Nat. Struct. Mol. Biol. 15, 1326-1333 (2008).

11. Hayashi, K., Ueno, H., Iino, R. \& Noji, H. Fluctuation theorem applied to $\mathrm{F}_{1}$-ATPase. Phys. Lett. 104, 218103 (2010).

12. Yasuda, R., Noji, H., Kinosita Jr, K. \& Yoshida, M. F $F_{1-}$ ATPase is a highly efficient molecular motor that rotates with discrete $120^{\circ}$ steps. Cell 93, 1117-1124 (1998).

13. Noji, H., Blad, D., Yasuda, R., Itoh, H., Yoshida, M. \& Kinosita $\mathrm{Jr}, \mathrm{K}$. Purine but not pyrimidine nucleotides support rotation of $\mathrm{F}_{1}$-ATPase. J. Biol. Chem. 276, 25480-25486 (2001).

14. Yasuda, R., Noji, H., Yoshida, M., Kinosita Jr, K. \& Itoh, H. Resolution of distinct rotational substeps by submillisecond kinetic analysis of $\mathrm{F}_{1}$-ATPase. Nature 410, 898-904 (2001).

15. Pänke, O., Cherepanov, D. A., Gumbiowski, K., Engelbrecht, S. \& Junge, W. Viscoelastic dynamics of actin filaments coupled to rotary F-ATPase: angular torque profile of the enzyme. Biophys. J. 81, 1220-1233 (2001).

16. Sowa, Y. \& Berry, R. M. Bacterial flagellar motor. Q. Rev. Biophys. 41, 103-132 (2008).

17. Harada, Y., Ohara, O., Takatsuki, A., Itoh, H., Shimamoto, N. \& Kinosita Jr, K. Direct observation of DNA rotation during transcription by Escherichia coil RNA polymerase. Nature 409, 113-115 (2001).

18. Imamura, H., Takeda, M., Funamoto, S., Shimabukuro, K., Yoshida, M. \& Yokoyama, K. Rotation scheme of $\mathrm{V}_{1}$-motor is different from that of $\mathrm{F}_{1}$-motor. Proc. Natl. Acad. Sci. USA 102, 17929-17933 (2005).

19. Usukura, E., Suzuki, T., Furuike, S., Soga, N., Saita, E., Hisabori, T., Kinosita Jr, K. \& Yoshida, M. Torque generation and utilization in the motor enzyme $\mathrm{F}_{0} \mathrm{~F}_{1}$-ATP synthase: half torque $\mathrm{F}_{1}$ with short-sized pushrod helix and reduced ATP synthesis by half-torque $\mathrm{F}_{0} \mathrm{~F}_{1}$. J. Biol. Chem. 287, 1885-1891 (2012).

20. Hayashi, K. \& Takagi, H. Fluctuation theorem applied to Dictyostelium discoideum systems. Phys. Soc. Jpn. 76, 105001 (2007). 